University of Nebraska - Lincoln

DigitalCommons@University of Nebraska - Lincoln

Anthony F. Starace Publications

Research Papers in Physics and Astronomy

February 2008

\title{
Threshold Phenomena in Electron-Atom Scattering in a Laser Field
}

\author{
N. L. Manakov \\ Voronezh State University, Universitetskaya pl. 1, Voronezh, 394006 Russia, manakov@phys.vsu.ru \\ Anthony F. Starace \\ University of Nebraska-Lincoln, astarace1@unl.edu
}

A. V. Flegel

Voronezh State University, Universitetskaya pl. 1, Voronezh, 394006 Russia

M. V. Frolov

Voronezh State University, Universitetskaya pl. 1, Voronezh, 394006 Russia

Follow this and additional works at: https://digitalcommons.unl.edu/physicsstarace

Part of the Physics Commons

Manakov, N. L.; Starace, Anthony F.; Flegel, A. V.; and Frolov, M. V., "Threshold Phenomena in Electron-Atom Scattering in a Laser Field" (2008). Anthony F. Starace Publications. 113.

https://digitalcommons.unl.edu/physicsstarace/113

This Article is brought to you for free and open access by the Research Papers in Physics and Astronomy at DigitalCommons@University of Nebraska - Lincoln. It has been accepted for inclusion in Anthony F. Starace Publications by an authorized administrator of DigitalCommons@University of Nebraska - Lincoln. 


\title{
Threshold Phenomena in Electron-Atom Scattering in a Laser Field
}

\author{
N. L. Manakov ${ }^{a}$, A. F. Starace ${ }^{b}$, A. V. Flegel $^{a}$, and M. V. Frolov ${ }^{a}$ \\ ${ }^{a}$ Voronezh State University, Universitetskaya pl. 1, Voronezh, 394006 Russia \\ e-mail:manakov@phys.vsu.ru \\ ${ }^{b}$ The University of Nebraska, Lincoln, NE 68588-0111, USA \\ Received November 19, 2007; in final form, December 13, 2007
}

\begin{abstract}
Effective-range theory is developed to describe the processes of multiphoton bremsstrahlung absorption and the emission of laser radiation that accompanies the scattering of electrons from atoms in a laser field. It is found that the cross sections for multiphoton absorption in the plateau region increases resonantly for electron energies corresponding to the thresholds of induced bremsstrahlung (i.e., multiples of the photon energy). It is shown that this effect is caused by the laser-field modified threshold phenomena in the cross sections for multichannel reactions (Baz' threshold anomalies).
\end{abstract}

PACS numbers: 03.65.Nk, 32.80.Wr, 34.80.Qb

DOI: $10.1134 / \mathrm{S} 0021364008020069$

Plateau effects (i.e., weak dependence of the cross sections for multiphoton processes on the number of absorbed photons $n$ in a wide range of $n$ values) are very interesting nonlinear phenomena in the interaction of a strong laser field with atoms and molecules. Plateau structures in the energy spectrum of photoelectrons in above-threshold ionization and in the spectrum of radiation appearing during the process of the generation of higher harmonics of a laser pump have been actively studied for more than a decade (e.g., see [1]). The presence of the plateau in the spectra of multiphoton bremsstrahlung absorption in electron-atom scattering was revealed for both linear [2] and circular [3] polarizations of the laser field by simulating the atomic potential $U(\mathbf{r})$ by the zero-range potential. Although the presence of the plateau allows one to observe experimentally high-energy electrons and higher harmonics, the absolute value of the $n$-photon cross sections in the plateau region is several orders of magnitude smaller than that in the region of small $n$ values. Therefore, it is important to seek mechanisms for increasing the cross sections in the plateau region. The cross sections for the processes of above-threshold ionization and the generation of higher harmonics can be increased by varying the intensity of the laser field near the threshold of the lowest multiphoton ionization channel. As shown in [46] for above-threshold ionization and in [5, 7] for the generation of higher harmonics for the example of an electron bound by short-range forces (negative ions), increases are caused by known threshold phenomena when one of the channels of a multichannel reaction is closed $[8,9]$ (see also a semiclassical interpretation of these phenomena in terms of the interference of classical electron trajectories in the laser field [10]).
Threshold phenomena can also occur in electronatom scattering in the laser field, because this process is substantially multichannel: the possible up transitions of the electron with momentum $p$ and energy $E=p^{2} / 2 m$ to states with energies $E_{n}=E+n \hbar \omega$ correspond to the absorption $(n>0)$, the elastic scattering $(n=0)$, and the induced emission $(n<0)$ of photons. In the last case, the number of emitted photons is limited by the threshold value $\left|n_{\min }\right|=[E / \hbar \omega]([x]$ is the integer part of $x)$. Hence, in contrast to above-threshold ionization and generation of higher harmonics, the threshold conditions for the scattering are independent of the field intensity and are achieved by varying the electron energy $E$ (or field frequency $\omega)$ so as to satisfy the relation $E=\mu \hbar \omega(\mu=$ $1,2, \ldots)$ corresponding to the multiphoton emission threshold with $n_{\min }=-\mu$. According to the general theory $[8,9]$, threshold anomalies can exist in all channels with $n>n_{\min }$. However, their character and the region of $n$ values in which the threshold modification of the cross sections is significant depend on the type of the process and the parameters of the problem and cannot be determined from the general consideration.

In this work, the effective-range theory [9] for the elastic scattering of electrons on the short-range potential $U(r)$ is generalized to the case of scattering in the presence of a laser field and is used to analyze threshold phenomena during the processes of multiphoton bremsstrahlung absorption and emission of laser photons. In such an approach, the electron-atom interaction is taken into account through the scattering length and effective range and the interaction of the electron with the laser field is precisely taken into account. It is shown that the scattering amplitude as a function of the 
energy has root branch points at the thresholds of the induced bremsstrahlung, the spectrum of scattered electrons exhibits all of four types of threshold anomalies described by Baz' [8], and the threshold effects are most significant in the plateau region for the multiphoton absorption process.

Scattering wavefunctions in the effective range theory. The wavefunction of the electron with energy $E$ and asymptotic momentum $\mathbf{p}$ in the potential $U(r)$ and light field with the electric vector $\mathbf{F}(t)=\mathbf{F} \cos \omega t$ has the form $\boldsymbol{\Psi}_{\mathbf{p}}(\mathbf{r}, t)=\exp (-i \epsilon t / \hbar) \boldsymbol{\Phi}_{\mathbf{p}}(\mathbf{r}, t)$, where $\epsilon=E+u_{p}$ is the quasi-energy, $u_{p}=e^{2} F^{2} / 4 m \omega^{2}$ is the average vibrational energy of the electron in the field, and the timeperiodic function $\boldsymbol{\Phi}_{\mathbf{p}}(\mathbf{r}, t)$ is a solution of Schrödinger equation

$$
\left[i \hbar \frac{\partial}{\partial t}+\epsilon+\frac{\hbar^{2}}{2 m} \Delta-U(r)-V(\mathbf{r}, t)\right] \boldsymbol{\Phi}_{\mathbf{p}}(\mathbf{r}, t)=0 .
$$

Here $V(\mathbf{r}, t)=|e|(\mathbf{r} \cdot \mathbf{F}(t))$ and

$$
\boldsymbol{\Phi}_{\mathbf{p}}(\mathbf{r}, t)=\chi_{\mathbf{p}}(\mathbf{r}, t)+\boldsymbol{\Phi}_{\mathbf{p}}^{(s c)}(\mathbf{r}, t),
$$

where the incident wave $\chi_{\mathbf{p}}(\mathbf{r}, t)$ is the periodic part of the known wavefunction $\psi_{\mathbf{p}}(\mathbf{r}, t)=\exp (-i \epsilon t / \hbar) \chi_{\mathbf{p}}(\mathbf{r}, t)$ of a free electron in the field and the asymptotic expression for the scattered wave $\boldsymbol{\Phi}_{\mathbf{p}}^{(\mathrm{sc})}(\mathbf{r}, t)$ determines the amplitude $\mathscr{A}_{n}\left(\mathbf{p}, \mathbf{p}_{n}\right)$ and scattering cross section:

$$
\begin{gathered}
\left.\boldsymbol{\Phi}_{\mathbf{p}}^{(\mathrm{sc})}(\mathbf{r}, t)\right|_{r \rightarrow \infty}=\sum_{n=n_{\min }}^{\infty} \mathscr{A}_{n} r^{-1} e^{i p_{n} r / \hbar-i n \omega t}, \\
d \sigma_{n} / d \Omega_{\mathbf{p}_{n}}=\left(p_{n} / p\right)\left|\mathscr{A}_{n}\left(\mathbf{p}, \mathbf{p}_{n}\right)\right|^{2},
\end{gathered}
$$

where $p_{n}=\sqrt{2 m(E+n \hbar \omega)}$.

For the short-range potential $U(r)$ (with the range $r \approx r_{c}$ ), the solution of Eq. (1) can be obtained with the use of a method similar to that used to calculate the quasi-stationary states of a weakly bound electron in a constant electric field [11, 12] and in the light-wave field [13]. Let $U(r)$ have a weakly bound state with the orbital angular momentum $l$ and energy $E_{0}=-\hbar^{2} \kappa^{2} / 2 m$, so that $r_{c} \ll \kappa^{-1}$. In this case, the following boundary condition for the wavefunction $\boldsymbol{\Phi}_{\mathbf{p}}(\mathbf{r}, t)$ in the range $r_{c} \lesssim r \ll \kappa^{-1}$ can be written (cf. [13]):

$$
\begin{gathered}
\int \boldsymbol{\Phi}_{\mathbf{p}}(\mathbf{r}, t) Y_{l, m}^{*}(\hat{\mathbf{r}}) d \Omega_{\hat{\mathbf{r}}} \\
\sim \sum_{s=-\infty}^{\infty}\left[r^{-l-1}+\ldots+B_{l}(\epsilon+s \hbar \omega) r^{l}\right] f_{s}^{(m)} e^{-i s \omega t},
\end{gathered}
$$

where $f_{s}^{(m)}$ is the Fourier coefficients of a certain periodic function $f_{\mathbf{p}}^{(m)}(t)=\sum_{s} f_{s}^{(m)} e^{-i s \omega t}$ that determines the projection of $\boldsymbol{\Phi}_{\mathbf{p}}(\mathbf{r}, t)$ onto the spherical function $Y_{l, m}(\hat{\mathbf{r}})$ and plays a central role in the further consider- ations and the coefficient $B_{l}(E)$ containing the scattering phase $\delta_{l}$ due to the potential $U(r)$ is parameterized in terms of the scattering length $a_{l}$ and effective range $r_{l}$ according to the effective range theory as

$$
\begin{gathered}
(2 l-1) ! !(2 l+1) ! ! B_{l}(E) \equiv k^{2 l+1} \cot \delta_{l}(k) \\
=-1 / a_{l}+r_{l} k^{2} / 2, \quad k^{2}=2 m E / \hbar^{2} .
\end{gathered}
$$

According to Eqs. (3) and (5), the scattered wave $\boldsymbol{\Phi}_{\mathbf{p}}^{(\mathrm{sc})}(\mathbf{r}, t)$ in Eq. (2) beyond the range of the potential $U(r)$ (for $r>r_{c}$ ) should contain divergent spherical waves at $r \longrightarrow \infty$ and have a $\sim r^{-l-1}$ singularity at $r \longrightarrow$ 0 . Similarly to [13], such a solution of Eq. (1) with $U(r)=0$ can be represented in terms of the retarded Green's function $G\left(\mathbf{r}, t ; \mathbf{r}^{\prime}, t^{\prime}\right)$ of a free electron in the field $\mathbf{F}(t)$ as

$$
\boldsymbol{\Phi}_{\mathbf{p}}(\mathbf{r}, t)=\chi_{\mathbf{p}}(\mathbf{r}, t)+\sum_{m^{\prime}=-l}^{l} \tilde{\boldsymbol{\Phi}}_{\mathbf{p}}^{\left(m^{\prime}\right)}(\mathbf{r}, t),
$$

where

$$
\begin{gathered}
\tilde{\mathbf{\Phi}}_{\mathbf{p}}^{\left(m^{\prime}\right)}(\mathbf{r}, t)=-\left(2 \pi \hbar^{2}\right) /(m \kappa) \int_{-\infty}^{t} d t^{\prime} e^{i \epsilon\left(t-t^{\prime}\right) / \hbar} \\
\times\left. f_{\mathbf{p}}^{\left(m^{\prime}\right)}\left(t^{\prime}\right) \mathscr{Y}_{l, m^{\prime}}\left(\nabla_{\mathbf{r}^{\prime}}\right) G\left(\mathbf{r}, t ; \mathbf{r}^{\prime}, t^{\prime}\right)\right|_{\mathbf{r}^{\prime}=0} .
\end{gathered}
$$

Here, the differential operator $\mathscr{Y}_{l, m}\left(\nabla_{\mathbf{r}}\right)$ is obtained from the spherical function $\mathscr{Y}_{l, m}(\mathbf{r})$ by the substitution $\mathbf{r} \longrightarrow \nabla_{\mathbf{r}}$. The equation for the function $f_{\mathbf{p}}^{(m)}(t)$ follows from the matching of the projection of function (7) onto the spherical function $Y_{l, m}(\hat{\mathbf{r}})$ with boundary condition (5) for small $r$ values.

Below, we present the results for the most interesting cases of $s$ - $(l=0)$ and $p$-wave $(l=1)$ scatterings corresponding to scattering from atoms forming negative ions with outer $s$ and $p$ electrons, respectively. In addition, we use the dimensionless quantities: the field amplitude $F$ is measured in units of $F_{0}=\sqrt{2 m\left|E_{0}\right|^{3}} /|e| \hbar$ and energy and frequency are measured in units of $\left|E_{0}\right|$ and $\left|E_{0}\right| / \hbar$, respectively.

$S$-wave scattering. In this case, the differential operator in Eq. (8) is absent and the result for $\boldsymbol{\Phi}_{\mathbf{p}}(\mathbf{r}, t)$ formally coincides with the result for the zero-range potential [2] with the difference that the equation for $f_{\mathbf{p}}(t) \equiv f_{\mathbf{p}}^{(m=0)}(t)$ contains the effective range $r_{0}$. It is convenient to represent this equation in the form of the system of the linear inhomogeneous algebraic equations for the Fourier coefficients $f_{s}$ of the function $f_{\mathbf{p}}(t)$, which separates into two uncoupled systems, the first 
for the coefficients $f_{s} \equiv f_{2 k+\delta}$ with even subscripts $s$ ( $\delta=$ $0)$ and the second for the coefficients with odd subscripts $s(\delta=1)$ :

$$
\zeta\left(E_{2 k+\delta}\right) f_{2 k+\delta}=c_{2 k+\delta}+\sum_{k^{\prime}=-\infty}^{\infty} M_{k, k^{\prime}}\left(E_{\delta}\right) f_{2 k^{\prime}+\delta},
$$

where $E_{p}=E+p \omega$ and

$$
\zeta(E)=-i \sqrt{E+u_{p}}-1+\frac{r_{0}}{2}\left(1+E+u_{p}\right) .
$$

The quantities $c_{2 k+\delta}$ in Eq. (9) are the Fourier coefficients of the function $c_{\mathbf{p}}(t) \equiv \chi_{\mathbf{p}}(\mathbf{r}=0, t)$ :

$$
c_{s}=i^{s} \mathscr{g}_{-s}\left(\frac{2 F p}{\omega^{2}} \cos \theta, \frac{u_{p}}{2 \omega}\right) \text {, }
$$

where $\mathscr{F}_{n}(\alpha, \beta)=\sum_{m=-\infty}^{\infty} J_{n+2 m}(\alpha) J_{m}(\beta)$ is the generalized Bessel function [14] and $\theta$ is the angle between $\mathbf{p}$ and $\mathbf{F}$. The matrix elements $M_{k, k^{\prime}}$ in Eq. (9) can be represented in terms of one-dimensional integrals of the Bessel functions (see [2]). However, to analyze the threshold phenomena, it is convenient to represent them in the form

$$
\begin{gathered}
M_{k, k^{\prime}}(E) \\
=i \delta_{k, k^{\prime}}\left[\sum_{n=-\infty}^{\infty} J_{n}^{2}\left(\frac{u_{p}}{2 \omega}\right) \sqrt{E+2(k+n) \omega}-\sqrt{\epsilon}\right] \\
+\frac{1}{T^{2}} \sum_{n=-\infty}^{\infty} \int_{0}^{T} d t \int_{0}^{T} d t^{\prime} e^{i\left[\left(k^{\prime}-k\right) \omega t-n \omega t^{\prime}+\frac{u_{p}}{\omega} \cos \omega t \sin \omega t^{\prime}\right]} \\
\times\left[\frac{e^{i x\left(t, t^{\prime}\right) \sqrt{E+\left(k+k^{\prime}+n\right) \omega}}-1}{x\left(t, t^{\prime}\right)}-i \delta_{k, k^{\prime}} \sqrt{E+2 k \omega}\right], \\
T=2 \pi / \omega, \quad x\left(t, t^{\prime}\right)=\frac{4 F}{\omega^{2}} \sin \frac{\omega t}{2} \sin \frac{\omega t^{\prime}}{2} .
\end{gathered}
$$

Expression (12) is obtained with the use of the quasienergy representation of the Green's function of the electron in the light field $[15,16]$ instead of the nonstationary Green's function $G$ in the expression for $\boldsymbol{\Phi}_{\mathbf{p}}(\mathbf{r}$, t) in Eq. (7).

With the known solution of the system of Eqs. (9) (which can generally be obtained only numerically), the function $\boldsymbol{\Phi}_{\mathbf{p}}(\mathbf{r}, t)$ given by Eqs. (7) and (8) is completely determined and its asymptotic expression (3) in the limit $r \longrightarrow \infty$ provides the $s$-wave scattering amplitude

$$
\mathscr{A}_{n}^{(s)}\left(\mathbf{p}, \mathbf{p}_{n}\right)=\sum_{s} i^{n-s} f_{s} \mathscr{\Phi}_{n-s}\left(\frac{2 F p_{n}}{\omega^{2}} \cos \theta_{n}, \frac{u_{p}}{2 \omega}\right),
$$

where $\theta_{n}$ is the angle between $\mathbf{p}_{n}$ and $\mathbf{F}$.
$\boldsymbol{P}$-wave scattering. Performing the differentiation in Eq. (8) for the Fourier coefficients $f_{s}^{\left(m^{\prime}\right)}$ of the function $f_{\mathbf{p}}^{\left(m^{\prime}\right)}(t)$ with $l=1$, one can obtain the system of linear equations that is similar to system (9). However, the results are lengthy and we present only the final expression for the amplitude

$$
\begin{aligned}
& \mathscr{A}_{n}^{(p)}\left(\mathbf{p}, \mathbf{p}_{n}\right)=i^{n-1} \sum_{m=-1}^{1} \sum_{s}(-i)^{s} f_{s}^{(m)} \\
\times & {\left[\mathscr{Y}_{1, m}\left(\mathbf{p}_{n}\right) \mathscr{J}_{n-s}\left(\frac{2 F p_{n}}{\omega^{2}} \cos \theta_{n}, \frac{u_{p}}{2 \omega}\right)\right.} \\
- & \left.\delta_{m 0} \frac{F}{\omega} \sqrt{\frac{3}{4 \pi}} \mathscr{\Phi}_{n-s, 1}\left(\frac{2 F p_{n}}{\omega^{2}} \cos \theta_{n}, \frac{u_{p}}{2 \omega}\right)\right],
\end{aligned}
$$

where $2 \mathscr{I}_{n, 1}(\alpha, \beta)=\mathscr{I}_{n+1}(\alpha, \beta)+\mathscr{F}_{n-1}(\alpha, \beta)$.

Analysis of the threshold phenomena. To analyze the dependence of the amplitude $\mathscr{A}_{n}^{(s)}\left(\mathbf{p}, \mathbf{p}_{n}\right)$ on the energy $E$ near the thresholds $E=\mu \omega$ of the $\mu$-photon emission, we first analyze the matrix element $M_{k, k}(E)$ in Eq. (12). It can be shown that the even terms of the expansion of the exponential including $E$ in the integrand in Eq. (12) do not contribute to the integrals with respect to $t$ and $t^{\prime}$. Thus, $M_{k, k^{\prime}}(E)$ is a square-root function of the energy $E$ and the first correction to the threshold value $M_{k, k^{\prime}}(E=\mu \omega)$ can be obtained by expanding the integrand in Eq. (12) in the powers of $(E-\mu \omega)^{1 / 2}$ with the subsequent analytical calculation of the integrals with respect to $t$ and $t^{\prime}$. As a result, the matrix element $\tilde{M}_{k, k^{\prime}}(E) \equiv M_{k, k^{\prime}}(E)-\delta_{k, k^{\prime}} \zeta(E)$ near $E=\mu \omega$ is given by the expression

$$
\begin{gathered}
\tilde{M}_{k, k^{\prime}}\left(E_{\delta}\right) \approx \tilde{M}_{k, k^{\prime}}((\mu+\delta) \omega)+i \sqrt{E-\mu \omega} \Delta \tilde{M}_{k, k^{\prime}}, \\
\Delta \tilde{M}_{k, k^{\prime}}=(-1)^{k-k^{\prime}} J_{k+\frac{\mu+\delta}{2}}\left(\frac{u_{p}}{2 \omega}\right) J_{k^{\prime}+\frac{\mu+\delta}{2}}\left(\frac{u_{p}}{2 \omega}\right) .
\end{gathered}
$$

As above, $\delta=0$ (1) for even (odd) $s$ and $s^{\prime}$ values and the parities of $\mu$ and $\delta$ should be the same. This means that the behavior of the amplitude $\mathscr{A}_{n}^{(s)}\left(\mathbf{p}, \mathbf{p}_{n}\right)$ near the threshold of the emission of an even (odd) number of photons is governed by the even (odd) Fourier coefficients $f_{2 k+\delta}$ of the function $f_{\mathbf{p}}(t)$.

The equations for the correction $\sim \sqrt{E-\mu \omega}$ to the threshold coefficients $f_{s}^{(\mu)} \equiv f_{s}(E=\mu \omega)$,

$$
\left.f_{s}(E)\right|_{E \rightarrow \mu \omega} \approx f_{s}^{(\mu)}+i \sqrt{E-\mu \omega} \Delta f_{s},
$$


are obtained by the substitution of Eqs. (15) and (16) into Eq. (9):

$$
\sum_{k^{\prime}} \tilde{M}_{k, k^{\prime}}((\mu+\delta) \omega) \Delta f_{2 k^{\prime}+\delta}=-\sum_{k^{\prime}} \Delta \tilde{M}_{k, k^{\prime}} f_{2 k^{\prime}+\delta}^{(\mu)}
$$

This system of equations for $\Delta f_{s}$ is significantly simplified in the low-frequency approximation, because the off-diagonal matrix elements $\tilde{M}_{k, k^{\prime}}$ can be neglected in this case [2]. Moreover, the threshold coefficients $f_{2 k^{\prime}+\delta}^{(\mu)}$ in this case can also be taken in the zeroth approximation [neglecting the off-diagonal matrix elements $M_{k, k^{\prime}}$ in Eq. (9)]:

$$
f_{2 k+\delta}^{(\mu)} \approx-c_{2 k+\delta}^{(\mu)} / \tilde{M}_{k, k}((\mu+\delta) \omega) .
$$

As a result, the correction $\Delta f_{s}$ is given by the expression

$$
\Delta f_{2 k+\delta} \approx \frac{1}{\tilde{M}_{k, k}((\mu+\delta) \omega)} \sum_{k^{\prime} \neq k} \frac{\Delta \tilde{M}_{k, k^{\prime}} c_{2 k^{\prime}+\delta}^{(\mu)}}{\tilde{M}_{k^{\prime}, k^{\prime}}((\mu+\delta) \omega)} .
$$

The scattering amplitude in Eq. (13), as well as $f_{s}(E)$ [see Eq. (16)], has the root branch points at the thresholds:

$$
\left.\mathscr{A}_{n}\left(\mathbf{p}, \mathbf{p}_{n}\right)\right|_{E \rightarrow \mu \omega} \approx \mathscr{A}_{n}^{(\mu)}+i \sqrt{E-\mu \omega} \Delta \mathscr{A}_{n},
$$

which lead to the threshold features in the cross sections $d \sigma_{n} / d \Omega_{\mathbf{p}_{n}}$ in the channels with various $n$ values according to the general theory of threshold phenomena $[8,9]$.

Numerical results and discussion. Figure 1a shows the spectrum of the scattered electrons for the energies near the threshold value $E=16 \hbar \omega$ for scattering from the hydrogen atom $\left(a_{0} \kappa=1.453, r_{0} \kappa=0.623\right.$, and the binding energy of the ion $H^{-}$is $\left|E_{0}\right|=0.755 \mathrm{eV}$ ) in the field of the $\mathrm{CO}_{2}$ laser with an intensity of $I=$ $1.44 \times 10^{11} \mathrm{~W} / \mathrm{cm}^{2}$. According to the figure, the cross sections $d \sigma_{n} / d \Omega_{\mathbf{p}_{n}}$ change strongly (by one order of magnitude) when the energy is changed by only $\pm 0.01 \mathrm{eV}$ near $E=16 \hbar \omega$ in the plateau region, whereas the threshold anomalies are absent for small $n$ values. The threshold phenomena are also small for induced emission processes (see Fig. 2), where they give rise to a noticeable change in the cross sections only in the two channels nearest to the closed channel. Figure 3 a shows the spectrum of $p$-wave scattering of electrons from the fluorine atom $\left[a_{1} \kappa^{3}=0.784, r_{1} \kappa^{-1}=-4.553\right.$, and $\left.\left|E_{0}\left(F^{-}\right)\right|=3.4 \mathrm{eV}\right]$ in the field with $I=2.2 \times 10^{12} \mathrm{~W} / \mathrm{cm}^{2}$ and $\hbar \omega=0.688 \mathrm{eV}$ for the energy $E \approx 17 \hbar \omega$. As compared to the $s$-wave scattering, the threshold increases in the cross sections are more pronounced and are more than an order of magnitude. For both $s$ - and $p$-wave scatterings, both increases and decreases in the cross sections are observed at the threshold energies in the

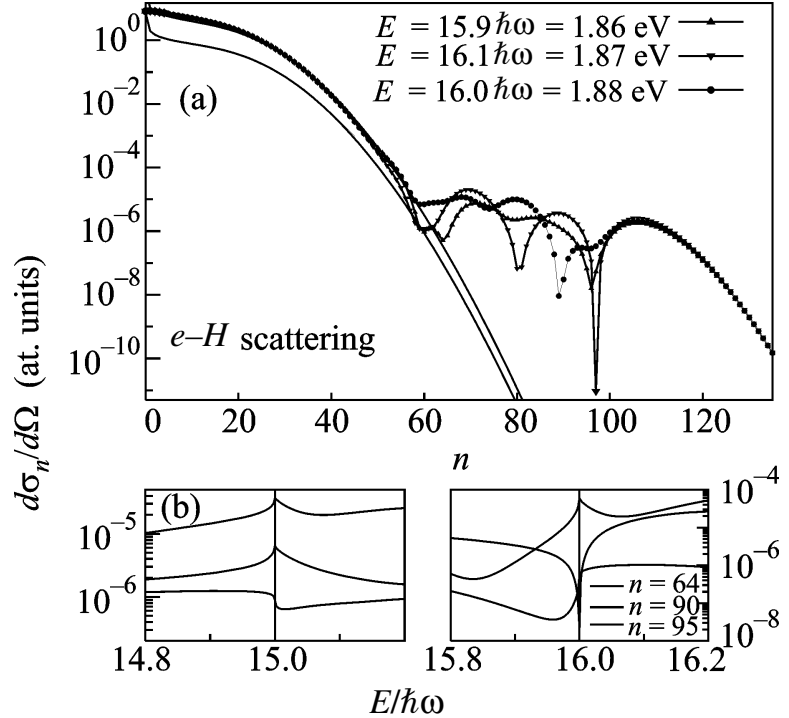

Fig. 1. Differential cross section $d \sigma_{n} / d \Omega$ for $e-H$ scattering at zero angle $\theta=\theta_{n}=0$ (along the direction of the linear polarization) for a $\mathrm{CO}_{2}$ laser with $\hbar \omega=0.117 \mathrm{eV}(\lambda=$ $10.6 \mu \mathrm{m}$ ) and intensity $I=1.44 \times 10^{11} \mathrm{~W} / \mathrm{cm}^{2}$. (a) The cross section versus the number of absorbed photons, $n$, for energies $E$ of the incident electron presented in the figure. The dotted and dashed lines are the results obtained by Bunkin and Fedorov [17] and Kroll and Watson [18]. (b) The cross section versus energy $E$ near the even $(\mu=16)$ and odd $(\mu=$ 15 ) thresholds of induced emission for a number of $n$ values presented in the figure.

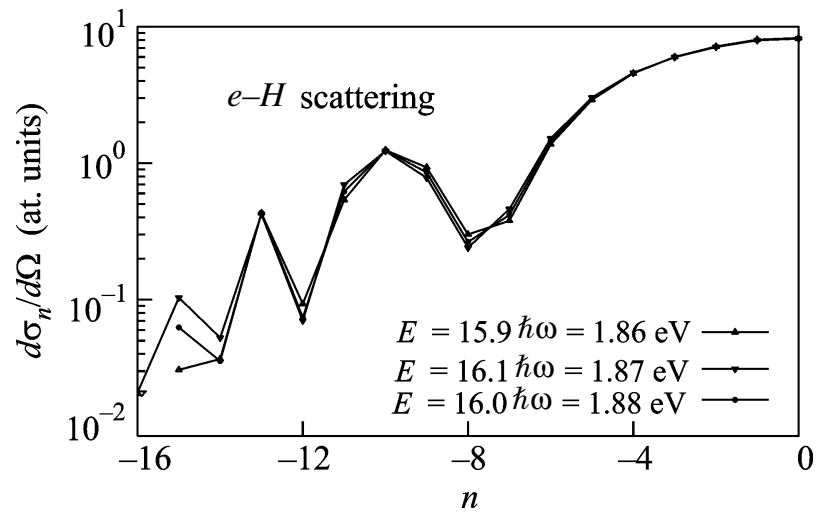

Fig. 2. Same as in Fig. 1a, but for induced bremsstrahlung in $e-H$ scattering.

plateau region for various $n$ values. This is due to the different energy dependences of the cross sections for different $n$ values near $E=\mu \omega$ : as seen in Figs. $1 \mathrm{~b}$ and $3 \mathrm{~b}$, this dependence can have the form of cusps or steps in agreement with the Baz' theory $[8,9]$.

To interpret the results presented in Figs. 1-3, note that, according to Eq. (19), the threshold modification 


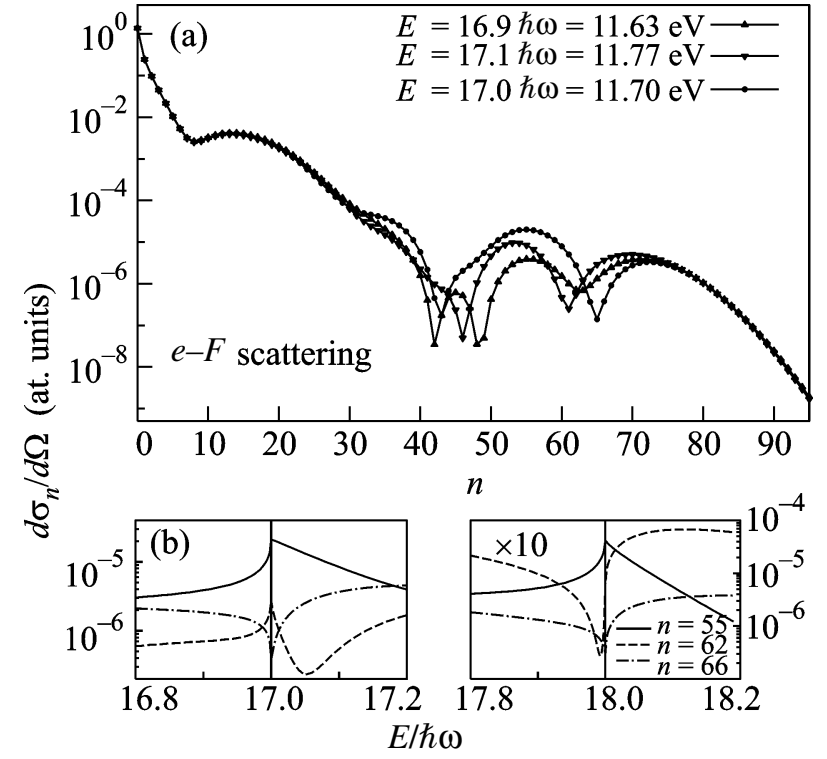

Fig. 3. Same as in Fig. 1, but for the $e-F$ scattering in the field with $I=2.2 \times 10^{12} \mathrm{~W} / \mathrm{cm}^{2}$ and $\hbar \omega=0.688 \mathrm{eV}$.

of the cross sections is most significant for $n$ values such that the regular part of the amplitude $\left(\mathscr{A}_{n}^{(\mu)}\right)$ is comparable with the correction $\sim \sqrt{E-\mu \omega}$. The quantity $\Delta \mathscr{A}_{n}$ is small for all $n$ values, because it is determined by small off-diagonal matrix elements $\Delta \tilde{M}_{k, k^{\prime}}$ in Eq. (18). In our approach, the off-diagonal matrix elements $M_{k, k^{\prime}}$ describe the effects of the coupling between the scattering channels with different numbers $n$ of absorbed/emitted photons. According to Eq. (9), these matrix elements describe the influence of the effects of higher orders in the interaction of the scattered electron with the atomic potential on the Fourier coefficients $f_{s}$ of the function $f_{\mathbf{p}}(t)$ and, hence, on the scattering amplitude with different $n$ values [according to Eqs. (13) and (14)]. Moreover, the plateau effects appear due to the interchannel coupling induced by the laser field and disappear when system (9) for the coefficients $f_{s}$ is solved in the diagonal approximation $M_{k, k^{\prime}} \approx M_{k, k} \delta_{k, k^{\prime}}$ [2]. The total contribution of these coefficients provides the scattering amplitudes $\mathscr{A}_{n}\left(\mathbf{p}, \mathbf{p}_{n}\right)$ that decrease rapidly as $n$ increases and describe only the low-energy part of the electron spectrum in Figs. 1a and 3a. As seen in Fig. 1a, this spectral range is also well approximated by the known Bunkin-Fedorov [17] and Kroll-Watson [18] formulas, where the effects of the coupling between channels are disregarded. ${ }^{1}$ Therefore, the scattering amplitude in the plateau region is determined

\footnotetext{
${ }^{1}$ Results [17] and [18] in Fig. 1a differ from each other, because the applicability condition for the Born approximation [17] $\left(\left|E_{0}\right| / E \approx 0.40\right)$ in this case is worse than the condition for the low-frequency approximation $[18]\left(\hbar \omega /\left|E_{0}\right| \approx 0.15\right)$.
}

only by small corrections to $f_{s}$ that originate from the off-diagonal matrix element $M_{k, k^{\prime}}$ in the system of Eqs. (9). As a result, the scattering cross section in the plateau region are several orders of magnitude smaller than those for small $n$ values and $\mathscr{A}_{n}^{(\mu)}$ and $\Delta \mathscr{A}_{n}$ in Eq. (19) become comparable, leading to an anomalously large threshold effect in this region.

The above results constitute a rare example of the semianalytical solution of the problem of multichannel scattering with the exact inclusion of the effects of the coupling between the channels. This example shows that the threshold phenomena accompanying the opening/closing of the lowest bremsstrahlung channel can anomalously change (by several orders of magnitude!) the scattering cross sections in the channels with $n$-photon absorption. Together with the results for abovethreshold ionization and the generation of higher harmonics [4-7], our analysis shows that threshold phenomena are typical for the plateau region in the cross sections for all atomic photoprocesses in a strong laser field. The threshold increases in the scattering cross sections appear at both even and odd thresholds of multiphoton emission, whereas the threshold anomalies in above-threshold ionization and the generation of higher harmonics appear only when an even (odd) channel of the multiphoton ionization from a bound state with the orbital angular momentum $l=0(1)$ is closed $[5,6]$.

We are grateful to M.V. Fedorov for a number of valuable remarks. This work was supported by the Russian Foundation for Basic Research (project no. 07-0200574) and the U.S. Department of Energy (grant no. DE-FG02-96ER14646). The work of A.V.F. was supported by the Council of the President of the Russian Federation for Support of Young Scientists and Leading Scientific Schools (project no. MK-8715.2006.2).

\section{REFERENCES}

1. W. Becker, F. Grabson, R. Kopold, et al., Adv. At. Mol. Opt. Phys. 48, 35 (2002).

2. N. L. Manakov, A. F. Starace, A. V. Flegel, and M. V. Frolov, Pis'ma Zh. Éksp. Teor. Fiz. 76, 316 (2002) [JETP Lett. 76, 258 (2002)].

3. A. V. Flegel, M. V. Frolov, N. L. Manakov, and A. F. Starace, Phys. Lett. A 334, 197 (2005).

4. B. Borca, M. V. Frolov, N. L. Manakov, and A. F. Starace, Phys. Rev. Lett. 88, 193001 (2002).

5. N. L. Manakov and M. V. Frolov, Pis'ma Zh. Éksp. Teor. Fiz. 83, 630 (2006) [JETP Lett. 83, 536 (2006)].

6. K. Krajewska, I. I. Fabrikant, and A. F. Starace, Phys. Rev. A 75, 053407 (2006).

7. B. Borca, A. F. Starace, A. V. Flegel, M. V. Frolov, and N. L. Manakov, Phys. Rev. A 65, 051402(R) (2002). 
8. A. I. Baz', Zh. Éksp. Teor. Fiz. 33, 923 (1957) [Sov. Phys. JETP 6, 709 (1958)]; A. I. Baz', Ya. B. Zel'dovich, and A. M. Perelomov, Scattering, Reactions and Decays in Nonrelativistic Quantum Mechanics (Nauka, Moscow, 1971, 2nd ed.; Israel Program for Scientific Translations, Jerusalem, 1966, transl. of the 1st Russ. ed.).

9. L. D. Landau and E. M. Lifshitz, Quantum Mechanics: Non-Relativistic Theory (Nauka, Moscow, 1989, 4th ed.; Pergamon Press, Oxford, 1977, 3rd ed.).

10. S. V. Popruzhenko, Ph. A. Korneev, S. P. Goreslavski, and W. Becker, Phys. Rev. Lett. 89, 023001 (2002).

11. Yu. N. Demkov and G. F. Drukarev, Zh. Éksp. Teor. Fiz. 81, 1218 (1981) [Sov. Phys. JETP 54, 650 (1981)].

12. S. P. Andreev, B. M. Karnakov, and V. D. Mur, Pis'ma Zh. Éksp. Teor. Fiz. 37, 155 (1983) [JETP Lett. 37, 187 (1983)].
13. M. V. Frolov, N. L. Manakov, E. A. Pronin, and A. F. Starace, Phys. Rev. Lett. 91, 053003 (2003).

14. A. I. Nikishov and V. I. Ritus, Zh. Éksp. Teor. Fiz. 46, 776 (1964) [Sov. Phys. JETP 19, 529 (1964)].

15. N. L. Manakov and A. G. Fainshtein, Teor. Mat. Fiz. 48, 375 (1981) [Theor. Math. Phys. 48, 815 (1982)].

16. M. V. Frolov, A. V. Flegel, N. L. Manakov, and A. F. Starace, Phys. Rev. A 75, 063408 (2007).

17. F. V. Bunkin and M. V. Fedorov, Zh. Éksp. Teor. Fiz. 49, 1569 (1965) [Sov. Phys. JETP 22, 844 (1965)].

18. N. M. Kroll and K. M. Watson, Phys. Rev. A 8, 804 (1973).

Translated by R. Tyapaev 